\section{Fingerprint and high-wavenumber Raman spectroscopy in a human-swine coronary xenograft in vivo}

\author{
Alexandra H. Chau, ${ }^{a, b}$ Jason T. Motz, \\ Joseph A. Gardecki, ${ }^{\text {b }}$ Sergio Waxman, ${ }^{\text {d,e }}$ \\ Brett E. Bouma, ${ }^{\mathrm{b}, \mathrm{f}, \mathrm{f}}$ and Guillermo J. Tearney ${ }^{\mathrm{b}, \mathrm{c}, \mathrm{f}, \mathrm{g}, *}$ \\ ${ }^{a}$ Massachusetts Institute of Technology, Department of \\ Mechanical Engineering, Cambridge, Massachusetts 02139 \\ ${ }^{b}$ Massachusetts General Hospital, Wellman Center for \\ Photomedicine, Boston, Massachusetts 02114 \\ ${ }^{\mathrm{C}}$ Harvard Medical School, Boston, Massachusetts 02115 \\ dLahey Clinic, Burlington, Massachusetts 01805 \\ 'Tufts University School of Medicine, Boston, Massachusetts \\ 02110 \\ fHarvard-MIT, Division of Health Sciences and Technology, \\ Cambridge, Massachusetts 02139 \\ ${ }^{\mathrm{g}}$ Massachusetts General Hospital, Department of Pathology, \\ Boston, Massachusetts 02114
}

\begin{abstract}
Intracoronary Raman spectroscopy could open new avenues for the study and management of coronary artery disease due to its potential to measure the chemical and molecular composition of coronary atherosclerotic lesions. We have fabricated and tested a 1.5-mm-diameter (4.5 Fr) Raman catheter capable of collecting Raman spectra in both the fingerprint $\left(400-1800 \mathrm{~cm}^{-1}\right)$ and high-wavenumber $\left(2400-3800 \mathrm{~cm}^{-1}\right)$ regions. Spectra were acquired in vivo, using a human-swine xenograft model, in which diseased human coronary arteries are grafted onto a living swine heart, replicating the disease and dynamic environment of the human circulatory system, including pulsatile flow and motion. Results show that distinct spectral differences, corresponding to the morphology and chemical composition of the artery wall, can be identified by intracoronary Raman spectroscopy in vivo. () 2008 Society of Photo-Optical Instrumentation Engineers. [DOI: 10.1117/1.2960015]
\end{abstract}

Keywords: Raman spectroscopy; intravascular catheter atherosclerosis; coronary artery; xenograft; fiber optic probe.

Paper 08085LR received Mar. 10, 2008; revised manuscript received May 9, 2008; accepted for publication May 12, 2008; published online Jul. 24, 2008.

Approximately one of every five deaths in the United States is caused by acute myocardial infarction (AMI). ${ }^{1}$ AMI is believed to result from certain types of coronary plaques that precipitate thrombus formation, subsequently occluding flow and causing ischemia and cell death. ${ }^{2}$ Autopsy studies have shown that these high-risk coronary lesions usually contain a fibrous cap (collagen, macrophages, lymphocytes), necrotic core (extracellular lipid, cholesterol crystals, necrotic debris), proteoglycans, or superficial calcifications. ${ }^{2,3}$ Raman spectroscopy in the fingerprint $(\mathrm{FP})$ region $\left(400-1800 \mathrm{~cm}^{-1}\right)$

*Tel: (617) 724-2979; E-mail: tearney@helix.mgh.harvard.edu can detect the majority of these components, ${ }^{4}$ and has been demonstrated for atherosclerotic plaque diagnosis ex vivo, ${ }^{5,6}$ as well as in large-diameter arteries in vivo.,8 Highwavenumber (HW) Raman spectroscopy (2400-3800 cm ${ }^{-1}$ ) has been recently investigated for arterial diagnosis ex vivo. ${ }^{9}$

Conducting Raman spectroscopy in human coronary arteries in vivo requires a small-diameter $(<1.5 \mathrm{~mm}$, the diameter of a typical coronary angioscope ${ }^{10}$ ) catheter that can be safely maneuvered within the coronary artery tree. The optical catheter is comprised of one or more optical fibers (typically fused silica) and distal optical elements to direct light to and collect light from the tissue. Laser light propagating through fused silica fiber generates a large Raman background signal that can mask the Raman signal from the tissue, particularly in the FP region. Thus, a FP Raman catheter requires at least two separately filtered channels: a shortpass-filtered excitation channel that blocks the fiber background and ensures the tissue is excited with laser light only, and a longpass-filtered collection channel that allows only the Raman component from the tissue to propagate back through the catheter to the Raman spectrometer.

The need for two separate channels with distinct optical filters at the catheter's distal tip presents a significant challenge for fabricating small-diameter, flexible Raman catheters suitable for intracoronary use. Several FP Raman probes have been demonstrated for atherosclerosis, all featuring a ring of collection fibers surrounding a central excitation fiber. ${ }^{7,8,11}$ With the exception of the micro-Raman probe developed by Komachi et al.," these devices have not been suitable for intracoronary Raman spectroscopy because they either were too large and inflexible to be introduced into human coronary arteries or have not had sufficient optical throughput.

HW Raman is advantageous compared with FP Raman in that fused silica fibers have significantly less background signal in the HW region, obviating the need for distal optical filters and allowing HW Raman spectra to be acquired through a single fiber. ${ }^{9,12}$ While FP and HW Raman signals provide complementary chemical information, ${ }^{9}$ the diagnostic potentials of both regions have not been fully explored. The development of a single catheter capable of collecting both FP and HW Raman spectra will facilitate the investigation of the chemical and molecular information content that can be obtained from each wavenumber region alone and in combination.

We have constructed a prototype Raman catheter capable of acquiring both FP and HW spectra. The catheter was comprised of an inner optical core surrounded by a transparent nylon sheath [Fig. 1]. The optical core contained a central excitation fiber surrounded by an outer ring of six collection fibers. Each fiber was made of low $\mathrm{OH}$, fused silica, with a $100-\mu \mathrm{m}$ core diameter (Polymicro Technologies; Phoenix, AZ). The catheter utilized a custom-manufactured, monolithic 775 - $\mu \mathrm{m}$-diameter dielectric interference filter on a fused silica substrate (BARR Associates; Westford, MA), featuring a circular shortpass filter (830-nm cutoff) surrounded by an annular longpass filter (860-nm cutoff). The filter was bonded with epoxy to the distal end of the fiber bundle. A $1.5-\mathrm{mm}$ $(4.5 \mathrm{~F})$ outer-diameter stainless steel tube was cut to provide a groove, which served as an optical window [Fig. 1(c)]. The fiber bundle/filter unit was inserted into the tube and bonded, along with a $45^{\circ}$ aluminum-coated rod mirror oriented to pro- 


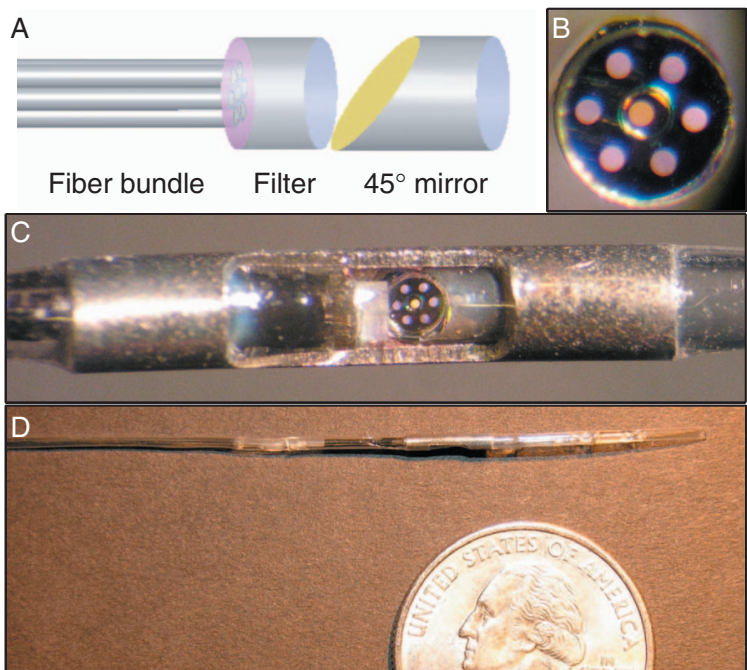

Fig. 1 (a) Schematic of the Raman catheter distal optics. (b) En face photograph of white light transmission through the fiber bundle and custom filter. The inner fiber (orange) was used for excitation; the six outer fibers (purple) were used for collection. (c) Close-up view of distal end of the Raman catheter. The mirror shows the reflection from the fiber bundle face. (d) Distal portion of the catheter.

vide lateral illumination and collection. Medical-grade heatshrink was used to seal the assembly, and the sheath was modified to provide a rapid-exchange guide wire port. When the catheter was held in contact with a sample, the laser spot size was approximately $1 \mathrm{~mm}$ in diameter. We believe the catheter's sampling volume in arterial tissue was approximately $1 \mathrm{~mm}^{3}$, as estimated from Monte Carlo simulations.

The collection fibers were coupled to a single spectrometer (Holospec f/1.8i, Kaiser Optical Systems, Inc.; Ann Arbor, MI) equipped with a CCD camera (Pixis 400BR, Princeton Instruments; Trenton, NJ). An 830-nm diode laser (Process Instruments Inc.; Salt Lake City, UT) and a 740-nm diode laser (Innovative Photonic Solutions; Monmouth Junction, $\mathrm{NJ}$ ) were coupled to the excitation fiber using a dichroic beamsplitter. Shutters were used to rapidly switch between the two wavelengths, allowing both FP (830-nm excitation) and HW (740-nm excitation) Raman spectra to be taken in rapid succession at a single site.

The prototype Raman catheter was tested in a humanswine xenograft model ${ }^{\dagger}$ in which a diseased human coronary is harvested from a cadaver, sutured on top of a living swine's heart to simulate cardiac motion, and then reconnected to the swine's arterial circulation to provide physiologic blood flow conditions. ${ }^{12,13}$ Following grafting, intracoronary devices can then be advanced through the grafted human artery. The human-swine xenograft accurately models the blood flow, motion, and compression and expansion of native coronary arteries, ${ }^{13}$ which enables testing of intracoronary devices for diagnosing real human pathology in an environment closely resembling human coronary physiology.

In accordance with the standard of care for catheterization procedures, heparin was administered to the swine to prevent

${ }^{+}$The human-swine xenograft procedure was approved by the Tufts-New England Medical Center's Institutional Animal Care and Use Committee (Protocol \#55-06). the formation of blood clots. After the graft was in place, Raman spectra were acquired from the grafted human artery in vivo, using illumination powers of $100 \mathrm{~mW}$ at $830 \mathrm{~nm}$ and $85 \mathrm{~mW}$ at $740 \mathrm{~nm}$. FP and HW spectra were acquired over 40 frames at $0.25 \mathrm{~s}$ each for a total acquisition time of $10 \mathrm{~s}$ at each site, which enabled us to evaluate spectral quality as a function of integration time. Based on previously reported results $^{7,8}$ and ex vivo experiments performed in our laboratory, ${ }^{14}$ we believe that these laser fluences do not cause arterial tissue damage or blood coagulation. After each Raman measurement, the interrogated site (identified by visualizing laser light transmitted through the artery wall) was marked with green ink for subsequent correlation with histology.

Raman spectra were extracted from the collected raw spectra using established postprocessing methods, ${ }^{8}$ including frame averaging, system spectral response correction, and catheter and fluorescence background removal. First, a specified number of raw data frames were averaged together and divided by a white light reference spectrum. The tissue fluorescence spectrum was removed by subtracting a fifth (FP) or sixth (HW) order polynomial, and the catheter background was removed by subtracting a processed and scaled background spectrum that was acquired while holding the catheter in air. To determine the minimum necessary acquisition time, we averaged various numbers of frames together and analyzed the resulting processed Raman spectra. In Fig. 2, we show Raman spectra that correspond to an integration time of $4 \mathrm{~s}$ (16 frames).

Raman spectra from the two sites differ significantly. In the first site [Figs. 2(a) and 2(b)], the FP Raman spectrum contains a prominent peak at $960 \mathrm{~cm}^{-1}$ (corresponding to the phosphate stretch of calcium hydroxyapatite) and minimal lipid. The Raman spectrum is consistent with histology, which demonstrates a large calcific nodule [Fig. 2(c)]. Spectra obtained at the second site [Figs. 2(d) and 2(e)] show peaks representative of adventitial triglycerides $\left(\mathrm{CH}_{2}\right.$ bends at 1301 and $1440 \mathrm{~cm}^{-1}$ and $\mathrm{C}=\mathrm{C}$ stretch at $1654 \mathrm{~cm}^{-1}$ in the $\mathrm{FP}$ spectrum $^{4}$ and $\sim 2836 \mathrm{~cm}^{-1}$ in the HW spectrum). There is an increased contribution from cholesterol $\left(\sim 2870 \mathrm{~cm}^{-1}\right)$ in the HW spectrum, which may correspond to a small amount of lipid staining seen on the Oil-red-O histology section [Fig. 2(f)]. A minute calcium hydroxyapatite signal is also present, which may result from the large, adjacent calcific nodule.

In the two samples shown, the arterial lumen is only marginally larger than the catheter diameter, and thus the catheter was nearly in contact with the lumen surface. We have performed ex vivo experiments suggesting that Raman spectra can be reliably obtained through a small distance of blood $(<0.5 \mathrm{~mm})$ and through a moderate distance of saline $(<2 \mathrm{~mm})$. Thus, in a larger-diameter artery, our Raman catheter may require a saline purge to clear blood and/or an additional mechanism to ensure contact with the arterial wall (e.g., a balloon).

In this letter, we have demonstrated a Raman catheter capable of acquiring both FP and HW Raman spectra in vivo. Compared to previously demonstrated fiber-based Raman probes, the design and fabrication of our catheter was simplified by the use of the single-piece, patterned dielectric filter module. While our current catheter has a relatively large outer diameter of $1.5 \mathrm{~mm}$, the limiting factor is the diameter of the fiber bundle and filter $(775 \mu \mathrm{m})$. Thus, the overall catheter 

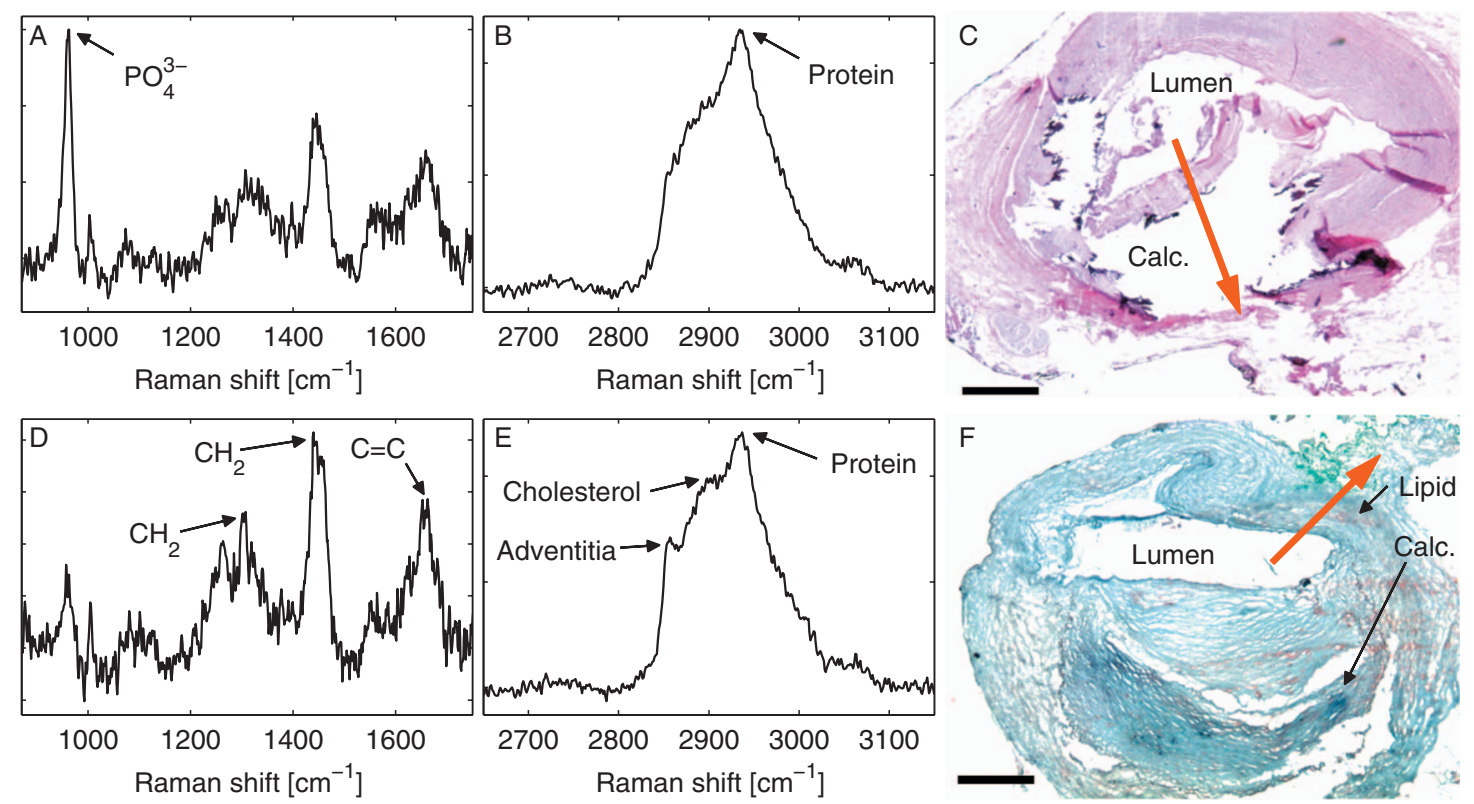

Fig. 2 Raman spectra obtained in a human artery grafted to the beating heart of a living swine. Spectra were processed from 16 averaged frames, representing a total acquisition time of $4 \mathrm{~s}$. (a) FP and (b) HW spectra obtained at Site \#1, with (c) corresponding hematoxylin and eosin histology. (d) FP and (e) HW spectra obtained at Site \#2, with (f) corresponding Oil-red-O histology. Black arrows denote brown-orange staining of lipid in the direction of the spectral measurement and dark blue staining of a large calcific nodule nearby. Red arrows in (c) and ( $\mathrm{f}$ ) denote spectral measurement locations and directions. Calc.—calcium hydroxyapatite. Scale bars, $0.5 \mathrm{~mm}$.

diameter can be reduced to less than $1 \mathrm{~mm}$ by simply replacing the side-viewing mirror and outer sheath, which will bring the catheter diameter on par with that of commercially available intracoronary imaging catheters. Future catheter development efforts will focus on reducing the catheter's diameter, scanning the inner optics with respect to the outer sheath, and increasing the Raman signal collection efficiency by improving the fiber bundle's filling density and optimizing the optics at the distal end of the catheter.

\section{Acknowledgments}

This study was supported by Prescient Medical, Inc. A.H.C. was supported by a Ruth L. Kirschstein individual fellowship (Grant no. F31EB007169) from the National Institute of Biomedical Imaging and Bioengineering.

\section{References}

1. "Heart Disease and Stroke Statistics—2008 Update," American Heart Association, Dallas, TX (2008).

2. R. Ross, "Atherosclerosis—an inflammatory disease," N. Engl. J. Med. 340(2), 115-126 (1999).

3. R. Virmani, A. P. Burke, A. Farb, and F. D. Kolodgie, "Pathology of the vulnerable plaque," J. Am. Coll. Cardiol. 47(C), C13-18 (2006).

4. H. P. Buschman, G. Deinum, J. T. Motz, M. Fitzmaurice, J. R. Kramer, A. van der Laarse, A. V. Bruschke, and M. S. Feld, "Raman microspectroscopy of human coronary atherosclerosis: Biochemical assessment of cellular and extracellular morphologic structures in situ," Cardiovasc. Pathol. 10(2), 69-82 (2001).

5. H. P. Buschman, J. T. Motz, G. Deinum, T. J. Römer, M. Fitzmaurice, J. R. Kramer, A. van der Laarse, A. V. Bruschke, and M. S. Feld, "Diagnosis of human coronary atherosclerosis by morphology-based Raman spectroscopy," Cardiovasc. Pathol. 10(2), 59-68 (2001).

6. T. J. Römer, J. F. Brennan, M. Fitzmaurice, M. L. Feldstein, G.
Deinum, J. L. Myles, J. R. Kramer, R. S. Lees, and M. S. Feld, "Histopathology of human coronary atherosclerosis by quantifying its chemical composition with Raman spectroscopy," Circulation 97(9), 878-885 (1998).

7. H. P. Buschman, E. T. Marple, M. L. Wach, B. Bennett, T. C. B. Schut, H. A. Bruining, A. V. Bruschke, A. van der Laarse, and G. J. Puppels, "In vivo determination of the molecular composition of artery wall by intravascular Raman spectroscopy," Anal. Chem. 72(16), 3771-3775 (2000).

8. J. T. Motz, M. Fitzmaurice, A. Miller, S. J. Gandhi, A. S. Haka, L. H. Galindo, R. R. Dasari, J. R. Kramer, and M. S. Feld, "In vivo Raman spectral pathology of human atherosclerosis and vulnerable plaque," J. Biomed. Opt. 11(2), 021003 (2006).

9. S. Koljenovic, T. C. B. Schut, R. Wolthuis, B. de Jong, L. Santos, P. J. Caspers, J. M. Kros, and G. J. Puppels, "Tissue characterization using high wave number Raman spectroscopy," J. Biomed. Opt. 10(3), 031116 (2005).

10. C. J. White, S. R. Ramee, T. J. Collins, A. E. Escobar, A. Karsan, D. Shaw, S. P. Jain, T. A. Bass, R. R. Heuser, P. S. Teirstein, R. Bonan, P. D. Walter, and R. W. Smalling, "Coronary thrombi increase PTCA risk: Angioscopy as a clinical tool," Circulation 93(2), 253258 (1996).

11. Y. Komachi, H. Sato, and H. Tashiro, "Intravascular Raman spectroscopic catheter for molecular diagnosis of atherosclerotic coronary disease," Appl. Opt. 45(30), 7938-7943 (2006).

12. J. T. Motz, J. Nazemi, S. Waxman, S. L. Houser, J. A. Gardecki, A. H. Chau, B. E. Bouma, J. F. Brennan, and G. J. Tearney, "Intracoronary Raman diagnostics in a human-to-porcine xenograft model," Am. J. Cardiol. 100(8A), 133L (2007).

13. S. Waxman, K. Khabbaz, R. Connolly, J. Tang, A. Dabreo, L. Egerhei, F. Ishibashi, J. E. Muller, and G. M. Tearney, "Intravascular imaging of atherosclerotic human coronaries in a porcine model: A feasibility study," Int. J. Card. Imaging 24(1), 37-44 (2008).

14. J. A. Gardecki, J. T. Motz, A. H. Chau, B. E. Bouma, and G. J. Tearney, "Establishing safe laser exposure levels for intracoronary Raman diagnostic procedures," (in preparation) (2008). 